\title{
Classes of Inverse Semirings and its Ordering
}

\author{
G. Rajeswari ${ }^{1}$, T. Vasanthi ${ }^{2}$ \\ Department of Applied Mathematics ${ }^{1,2}$, Yogi Vemana University ${ }^{1,2}$, Kadapa, Andhra Pradesh, India \\ Email:grajeswariyvu@gmail.com ${ }^{1}$,vasanthi_thatimakula@yahoo.co.in ${ }^{2}$
}

\begin{abstract}
In this paper we study the conditions under which an additively inverse semiring is a band and commutative. Also in the case of totally ordered additive inverse semiring in which $(S,+)$ is positively totally ordered, we prove the additive structure is $a+b=b+a=\max (a, b)$ for all $a, b$ in $S$. We have also framed an example for this result by considering 4 elements.
\end{abstract}

Index Terms - Additive inverse semiring; zero-square semiring; positively totally ordered semiring.

\section{INTRODUCTION}

Additive and multiplicative structures play an important role in determining the structure of semiring. If the multiplicative structure of a semiring is a rectangular band, then its additive structure is a band. Karvellas [4] proved that if the additively inverse semiring contains right multiplicative identity, then its additive structure is commutative. In [9] Zeleznekow studied additive inverse semirings and examined the conditions under which $(S,+)$ is a semilattice. In this paper we study the conditions under which $(S,+)$ is a commutative band and determine the structure of additively inverse semiring [4], [8] if $(S,+)$ is positively totally ordered [1],[2], [5],[6].

\section{PRELIMINARIES}

In this section, we have given some important definitions that are used in the theorems which are stated and proved in the following sections.

\section{Definition 2.1}

A triple $(S,+$,$) is called a semiring if (S,+)$ is a semigroup; $(S, \cdot)$ is a semigroup; $a(b+c)=a b+a c$ and $(b+c) a=b a+c a$ for every $a, b, c$ in $S$. The two operations + and $\cdot$ are called additive and multiplicative operations respectively.

\section{Definition 2.2}

A semiring $(S,+, \cdot)$ is called an additive inverse semiring if $(S,+)$ is an inverse semigroup that is for each $a \in S$ there exists a unique element $a^{\prime} \in S$ such that $a+a^{\prime}+a=a$ and $a^{\prime}+a+a^{\prime}=a^{\prime}$.

\section{Definition 2.3}

In a totally ordered semiring $(S,+, \cdot, \leq)$

(i) $(S,+, \leq)$ is positively totally ordered (p.t.o), if $a+x \geq a, x$ for all $a, x$ in $S$.

(ii) $(S, \cdot, \leq)$ is positively totally ordered (p.t.o), if $a x \geq a, x$ for all $a, x$ in $S$.

(iii) $(S,+, \leq)$ is negatively totally ordered (n.t.o), if $a+x \leq a, x$ for all $a, x$ in $S$.

(iv) $(S, \cdot, \leq)$ is negatively totally ordered (n.t.o) if $a x \leq a, x$ for all $a, x$ in $S$.

\section{Definition 2.4}

A semiring $\mathrm{S}$ is Positive Rational Domain (PRD) if $(S, \cdot)$ is an abelian group.

\section{Definition 2.5}

Zeroid of a semiring $(S,+, \cdot)$ is the set of all $\mathrm{x}$ in $\mathrm{S}$ such that $x+y=y$ or $y+x=y$ for some $\mathrm{y}$ in S. we may also term this as the zeroid of $(S,+)$ and it is denoted by Zd.

\section{Definition 2.6}

A semiring $\mathrm{S}$ with multiplicative zero is said to be a zero square semiring if $a^{2}=0$ for all $a$ in $S$.

\section{Definition 2.7}

A semigroup $(S,+)$ is commutative if $a+x=x+a$ for all $a, x$ in $S$.

A semigroup $\left(S,{ }^{-}\right)$is commutative if $a x=x a$ for all $a, x$ in $S$.

Definition 2.8 


\section{Available online at $w w w . i j r a t . o r g$}

A semigroup $(S,+)$ is additively idempotent if $a+a=a$ for all $a$ in $S$.

\section{Definition 2.9}

An element a in a semigroup $(S,+)$ is periodic if $m a=n a$ where $m$ and $n$ are positive integers. A semigroup $(S,+)$ is periodic if every one of its elements is periodic.

\section{Definition 2.10}

A semigroup $(S,+)$ is band if $a+a=a$ for all $a$ in $S$

A semigroup $(S, \cdot)$ is band if $a^{2}=a$ for all $a$ in $S$

\section{Definition 2.11}

A semiring $S$ is said to be mono semiring if $a+b=a b$ for all $a, b$ in $S$.

Note: Undefined concepts refer [3]

\section{STRUCTURE OF ADDITIVE INVERSE SEMIRINGS}

Theorem 3.1. Let $(S,+, \cdot)$ be an additively inverse PRD. Then $(S,+)$ is a commutative band.

Proof. Let $a \in S$. Then there exists $a^{\prime} \in S$

Such that $a+a^{\prime}+a=a$

$$
\begin{aligned}
& \Rightarrow a+a^{\prime}+a+a^{\prime}=a+a^{\prime} \\
& \Rightarrow\left(a+a^{\prime}\right) \cdot 1+\left(a+a^{\prime}\right) \cdot 1=a+a^{\prime} \\
& \Rightarrow\left(a+a^{\prime}\right)(1+1)=\left(a+a^{\prime}\right) \cdot 1 \\
& \Rightarrow 1+1=1, \text { since }(S, \cdot) \text { is a group. } \\
& \Rightarrow b(1+1)=b .1 \text { for all } b \in S \\
& \Rightarrow b+b=b
\end{aligned}
$$

Therefore $(S,+)$ is a band

Similarly by taking $a^{\prime}+a+a^{\prime}=a^{\prime}$, we can prove that $(S,+)$ is a band.

Using theorem $4(1)$ of [4], $(S,+)$ is a commutative. Hence $(S,+)$ is commutative band.

Theorem 3.2. Let $(S,+, \cdot)$ be an additive inverse semiring. Then the zeroid of $(S,+)=Z d=\left\{a+a^{\prime} / a \in S\right\}$ is a multiplicative ideal.

Proof. $a=a+\left(a^{\prime}+a\right)$ also $a^{\prime}=a^{\prime}+\left(a+a^{\prime}\right)$ Therefore $a^{\prime}+a$ and $a+a^{\prime} \in Z d$
Now we prove that $Z d$ is a multiplicative ideal.

Let $s \in S$

$a=a+a^{\prime}+a$ implies

$s a=s a+s a^{\prime}+s a$

$=s a+s\left(a^{\prime}+a\right)$

$\Rightarrow s\left(a^{\prime}+a\right) \in Z d$

Also $a^{\prime}=a^{\prime}+a+a^{\prime}$ implies

$s a^{\prime}=s a^{\prime}+s a+s a^{\prime}$

$=s a^{\prime}+s\left(a+a^{\prime}\right)$

$\Rightarrow s\left(a+a^{\prime}\right) \in Z d$

Similarly $\left(a+a^{\prime}\right) s \in Z d$

Therefore $Z d$ is a multiplicative ideal.

Theorem 3.3. Let $S$ be an additive inverse semiring. If $(S, \cdot)$ is a band, then $a a^{\prime}$ and $a^{\prime}$ a are additively periodic elements.

Proof. Given that $S$ is an additive inverse semiring $a+a^{\prime}+a=a \Rightarrow a^{2}+a a^{\prime}+a^{2}=a^{2}$

since $(S, \cdot)$ is a band then $a^{2}=a, a^{\prime 2}=a^{\prime}$ for all $a \in S$

$a+a a^{\prime}+a=a \Rightarrow a a^{\prime}+a a^{\prime 2}+a a^{\prime}=a a^{\prime}$

$\Rightarrow a a^{\prime}+a a^{\prime}+a a^{\prime}=a a^{\prime} \Rightarrow 3 a a^{\prime}=a a^{\prime}$

Thus $a a^{\prime}$ is additive periodic

Also $a^{\prime}+a+a^{\prime}=a^{\prime} \Rightarrow a^{\prime 2}+a^{\prime} a+a^{\prime 2}=a^{\prime 2}$

$\Rightarrow a^{\prime}+a^{\prime} a+a^{\prime}=a^{\prime} \Rightarrow a^{\prime} a+a^{\prime} a^{2}+a^{\prime} a=a^{\prime} a$

$\Rightarrow a^{\prime} a+a^{\prime} a+a^{\prime} a=a^{\prime} a \Rightarrow 3 a^{\prime} a=a^{\prime} a$

Thus $a^{\prime} a$ is additive periodic.

Theorem 3.4. If $\mathrm{S}$ is an additive inverse semiring and $(s, \cdot)$ is rectangular band, then following identities are true.

(i) $a+a^{\prime} a^{2}+a=a$

(ii) $a a^{\prime}+a^{\prime}+a a^{\prime}=a a^{\prime}$

(iii) $a^{\prime 2} a+a^{\prime} a^{2}+a^{\prime 2}+a=a^{\prime 2} a$

Proof. Given that $\mathrm{S}$ is an additive inverse semiring $a+a^{\prime}+a=a$

$a^{2}+a^{\prime} a+a^{2}=a^{2}$

$a^{3}+a^{\prime} a^{2}+a^{3}=a^{3}$

Since $(s, \cdot)$ is rectangular band

$a+a^{\prime} a^{2}+a=a$

By using above condition (i)

$\Rightarrow a a^{\prime}+a^{\prime} a^{2} a^{\prime}+a a^{\prime}=a a^{\prime}$

$a a^{\prime}+a^{\prime}+a a^{\prime}=a a^{\prime}$

By using above condition (i)

$\Rightarrow a^{\prime} a+a^{\prime 2} a^{2}+a^{\prime} a=a^{\prime} a$

$a^{\prime 2} a+a^{\prime 3} a^{2}+a^{\prime 2} a=a^{\prime 2} a$ 


\section{STRUCTURE OF TOTALLY ORDERED SEMIRINGS}

Theorem 4.1. If $S$ is a totally ordered additive inverse semiring and $(S,+)$ is positively totally ordered (p.t.o), then

(i) $a=a^{\prime}$ for all $a \in S$

(ii) $(S,+)$ is a band.

(iii) $a+b=b+a=\max (a, b)$.

Proof. $(i)$ Since $S$ is an additive inverse semiring for each $a \in S$ there exists $a^{\prime} \in S$ such that $a=a+a^{\prime}+a \geq a^{\prime}$, since $(S,+)$ is p.t.o

Also $a^{\prime}=a^{\prime}+a+a^{\prime} \geq a+a^{\prime} \geq a$

Using (1) and (2) $a=a^{\prime}$ for all $a \in S$.

(ii) $a=a+a^{\prime}+a \geq a+a^{\prime} \geq a$

since $(S,+)$ is p.t.o

Therefore $a=a+a^{\prime}$

Now $a=a+a^{\prime}+a=a+a$ using (3)

Hence $(S,+)$ is a band.

(iii) Let $a, b \in S$

Suppose $a<b$

Then $a+a \leq a+b \leq b+b$

$\Rightarrow a \leq a+b \leq b$

since $(S,+)$ is a band.

From (4) $a+b \leq b$

Since $(S,+)$ is p.t.o, $a+b \geq b$

From (5) and (6) $a+b=b=\max (a, b)$

Also $a<b$ implies $a+a \leq b+a \leq b+b$

$\Rightarrow a \leq b+a \leq b$

Since $(S,+)$ is p.t.o, $b+a \geq b$

From (7) and (8) $b+a=b$.

Similarly we can prove that $a+b=b+a=\max (a, b)$ if $b<a$.

Theorem 4.2. If $S$ is a totally ordered multiplicative inverse semiring and $(S, \cdot)$ is positively totally ordered, then

(i) $a=a^{*}$ for all $a \in S$

(ii) $(S, \cdot)$ is a band.

(iii) $a b=b a=\max (a, b)$.
Proof. (i) Since $S$ is an additive inverse semiring for each $a \in S$ there exists $a^{\prime} \in S$ such that

$a=a a^{*} a$ and $a=a^{*} a a^{*}$

now $a=a a^{*} a \geq a a^{*} \geq a^{*}$, since $(S, \cdot)$ is p.t.o.

also $a^{*}=a^{*} a a^{*} \geq a^{*} a \geq a$

using (9) and (10) $a=a^{*}$ for all $a \in S$

(ii) $a=a a^{*} a=a a=a^{2}$

hence $(S, \cdot)$ is a band

(iii) $a, b \in S$

Suppose $a<b$

then $a^{2} \leq a b \leq b^{2}$

$\Rightarrow a \leq a b \leq b$ since $(S, \cdot)$ is a band

$a b \geq b$

from (11) and (12) $a b=b=\max (a, b)$

also $b a=b=\max (a, b)$

similarly we can prove $a b=b a=\max (a, b)$ if $b<a$

Proposition 4.3. Let $(S,+, \cdot)$ is doubly inverse semiring. If $(S,+)$ and $(S, \cdot)$ are positively totally ordered, then $a+b=b+a=a b=b a=\max (a, b)$ and hence $S$ is mono semiring.

Proof. In the above theorem $a+b=b+a=\max (a, b)$

Since $(S, \cdot)$ is an inverse semiring and p.t.o.

$a=a a^{\prime} a \geq a a^{\prime} \geq a$

Also $a^{\prime}=a^{\prime} a a^{\prime} \geq a^{\prime} a \leq a$

From (13) and (14) $a=a^{\prime}$

Also $a=a a^{\prime} a \geq a a^{\prime} \geq a$

Therefore $a=a a^{\prime}=a^{2}$ since $a^{\prime}=a$

Suppose $a<b$

$\Rightarrow a^{2} \leq a b \leq b^{2} \Rightarrow a \leq a b \leq b$

Since $S$ is p.t.o $a b \geq b$

From (15) and (16) $a b=b=\max (a, b)$

Then if $b<a$, we compare that $a b=a=\max (a, b)$

Theorem 4.4. Let $(S,+, \cdot)$ be a totally ordered additive inverse semiring. If $S$ contains the multiplicative identity, then $(S,+)$ is a commutative band.

Proof. Using proposition 1 of [7] 


\section{Available online at www.ijrat.org}

$(S,+)$ is non-negatively ordered or non-positively ordered.

Suppose $(S,+)$ is non-negatively ordered

$a=a+a^{\prime}+a=a+a+a^{\prime} \geq a+a^{\prime}$ by using theorem 4 (1) of [ 4]

Also $a^{\prime}=a^{\prime}+a+a^{\prime}=a+a^{\prime}+a^{\prime} \geq a+a^{\prime}$

$a+a^{\prime} \leq a$ and $a+a^{\prime} \leq a^{\prime}$

From (11) $a+a^{\prime} \leq a$

$\Rightarrow a^{\prime}+a+a^{\prime} \leq a^{\prime}+a=a+a^{\prime}$, since $(S,+)$ is

commutative

$\Rightarrow a^{\prime} \leq a+a^{\prime}$

From (17) and (18), $\quad a+a^{\prime}=a$

$a=a+a^{\prime}+a=a+a$

Therefore $(S,+)$ is a band.

Similarly we can prove the theorem if $(S,+)$ is nonpositively ordered.

Example 4.5. The following example $\{i, j, k, l\}$ with $l<k<j<i$ satisfies the conditions of theorem 4.1 in which $(S,+)$ is p.t.o and $S$ is also a mono semiring. This example also satisfies the conditions of theorem 4.4.

\begin{tabular}{|c|c|c|c|c|}
\hline+ & $\mathrm{i}$ & $\mathrm{j}$ & $\mathrm{k}$ & $\mathrm{l}$ \\
\hline $\mathrm{i}$ & $\mathrm{i}$ & $\mathrm{j}$ & $\mathrm{k}$ & $\mathrm{l}$ \\
\hline $\mathrm{j}$ & $\mathrm{j}$ & $\mathrm{j}$ & $\mathrm{k}$ & $\mathrm{l}$ \\
\hline $\mathrm{k}$ & $\mathrm{k}$ & $\mathrm{k}$ & $\mathrm{k}$ & $\mathrm{l}$ \\
\hline $\mathrm{l}$ & $\mathrm{l}$ & $\mathrm{l}$ & $\mathrm{k}$ & $\mathrm{l}$ \\
\hline
\end{tabular}

\begin{tabular}{|c|c|c|c|c|}
\hline$\cdot$ & $\mathrm{i}$ & $\mathrm{j}$ & $\mathrm{k}$ & $\mathrm{l}$ \\
\hline $\mathrm{i}$ & $\mathrm{i}$ & $\mathrm{j}$ & $\mathrm{k}$ & $\mathrm{l}$ \\
\hline $\mathrm{j}$ & $\mathrm{j}$ & $\mathrm{j}$ & $\mathrm{k}$ & $\mathrm{l}$ \\
\hline $\mathrm{k}$ & $\mathrm{k}$ & $\mathrm{k}$ & $\mathrm{k}$ & $\mathrm{l}$ \\
\hline $\mathrm{l}$ & $\mathrm{l}$ & $\mathrm{l}$ & $\mathrm{l}$ & $\mathrm{l}$ \\
\hline
\end{tabular}

Theorem 4.6. If $S$ satisfies the conditions of theorem 4.1 and $S$ is a zero square semiring with additive identity 0 , then $S^{2}=\{0\}$.

Proof. By (iii) of theorem 4.1

$a+b=b+a=\max (a, b)$

Suppose $a<b$

Then $a+b=b+a=b$ $a+b=b$ implies

$\Rightarrow(a+b) b=b \cdot b$

$\Rightarrow a b+b^{2}=b^{2}$

$\Rightarrow a b+0=0$

Therefore $a b=0$

Also $b(a+b)=b \cdot b$

$\Rightarrow b a+b^{2}=b^{2}$

$\Rightarrow b a+0=0$

Therefore $b a=0$

Hence $S^{2}=\{0\}$

Example 4.7. The below tables satisfies the conditions of the theorem 4.6 with $m<l<k<j<i$.

\begin{tabular}{|c|c|c|c|c|c|}
\hline+ & $\mathrm{i}$ & $\mathrm{j}$ & $\mathrm{k}$ & $\mathrm{l}$ & $\mathrm{m}$ \\
\hline $\mathrm{i}$ & $\mathrm{i}$ & $\mathrm{i}$ & $\mathrm{i}$ & $\mathrm{i}$ & $\mathrm{i}$ \\
\hline $\mathrm{j}$ & $\mathrm{i}$ & $\mathrm{j}$ & $\mathrm{j}$ & $\mathrm{j}$ & $\mathrm{j}$ \\
\hline $\mathrm{k}$ & $\mathrm{i}$ & $\mathrm{j}$ & $\mathrm{k}$ & $\mathrm{k}$ & $\mathrm{k}$ \\
\hline $\mathrm{l}$ & $\mathrm{i}$ & $\mathrm{j}$ & $\mathrm{k}$ & $\mathrm{l}$ & $\mathrm{l}$ \\
\hline $\mathrm{m}$ & $\mathrm{i}$ & $\mathrm{j}$ & $\mathrm{k}$ & $\mathrm{l}$ & $\mathrm{m}$ \\
\hline
\end{tabular}

\begin{tabular}{|c|c|c|c|c|c|}
\hline$\cdot$ & $\mathrm{i}$ & $\mathrm{j}$ & $\mathrm{k}$ & $\mathrm{l}$ & $\mathrm{m}$ \\
\hline $\mathrm{i}$ & $\mathrm{m}$ & $\mathrm{m}$ & $\mathrm{m}$ & $\mathrm{m}$ & $\mathrm{m}$ \\
\hline $\mathrm{j}$ & $\mathrm{m}$ & $\mathrm{m}$ & $\mathrm{m}$ & $\mathrm{m}$ & $\mathrm{m}$ \\
\hline $\mathrm{k}$ & $\mathrm{m}$ & $\mathrm{m}$ & $\mathrm{m}$ & $\mathrm{m}$ & $\mathrm{m}$ \\
\hline $\mathrm{l}$ & $\mathrm{m}$ & $\mathrm{m}$ & $\mathrm{m}$ & $\mathrm{m}$ & $\mathrm{m}$ \\
\hline $\mathrm{m}$ & $\mathrm{m}$ & $\mathrm{m}$ & $\mathrm{m}$ & $\mathrm{m}$ & $\mathrm{m}$ \\
\hline
\end{tabular}

Theorem 4.8. Let $(S,+, \cdot)$ be a totally ordered additively inverse semiring. If $(S, \cdot)$ is positively totally ordered, then the following are true.

(i) $(a b)^{\prime} \geq a$ and $(a b)^{\prime} \geq b$

(ii) $a b \geq a^{\prime}$ and $a b \geq b^{\prime}$

(iii) $(a+b)^{\prime} \leq(a+a) b$

(iv) $(a+b)^{\prime} \leq a b$ if $(S,+)$ is a band.

Proof.

(i) $(a b)^{\prime}=a b^{\prime} \geq a$, since $(S, \cdot)$ is p.t.o. $(a b)^{\prime}=a^{\prime} b \geq b$, since $(S, \cdot)$ is p.t.o. 


\section{Available online at www.ijrat.org}

(ii) $a b=a^{\prime} b^{\prime} \geq a^{\prime}$ and $a b=a^{\prime} b^{\prime} \geq b^{\prime}$, since $(S, \cdot)$ is p.t.o.

(iii) $(a+b)^{\prime}=b^{\prime}+a^{\prime} \leq a b+a b=(a+a) b$, using (ii)

(iv) From (iii) $(a+b)^{\prime} \leq(a+a) b$ if $(S,+)$ is a band

$a+a=a$ hence $(a+b)^{\prime} \leq a b$.

\section{REFERENCES}

[1] Amala, M; Sulochana, N; Vasanthi, T. (2016): Classes of Regular Semiring. IOSR Journal of Mathematics, 12(5-1), pp. 70-71.

[2] Amala, M; Vasanthi, T. (2015): Idempotent property of Semirings. Journal of Pure Algebra, 5 (9), pp. 156-159.

[3] Jonathan, S; Golan. Semirings and their Applications Kluwer Academic Publisheres.

[4] Karvellas, P.H. (1974): Inverse Semirings. J. Austral. Math. Soc. 18, pp. 277-288

[5] Satyanarayana, M. (1979): Positively Ordered Semigroups. Lecture notes in Pure and Applied Mathematics, Marcel Dekker, Inc., Vol.42

[6] Satyanarayana, M. (1981): On the additive semigroup structure of Semirings. Semigroup Forum, Vol. 23, pp. 7-14.

[7] Satyanarayana, M. (1985): On the additive semigroup of ordered Semirings. Semigroup Forum, Vol. 31, pp. 193-199.

[8] Sen, M.K; Maity, S.K. (2006): Regular additively inverse semirings. Acta Math. Univ. Comenianac, Vol. LXXV, (1), pp.137-146.

[9] Zeleznekow, J. (1981): Regular Semirings, Semigroup Forum, Vol. 23, pp. 119-136. 